\title{
Functional Optimization in Complex Excitable Networks
}

\author{
Samuel Johnson, J. Marro, and Joaquín J. Torres \\ Departamento de Electromagnetismo y Física de la Materia, and In- \\ stitute "Carlos I" for Theoretical and Computational Physics, \\ Facultad de Ciencias, Universidad de Granada, 18071-Granada, Spain.
}

\begin{abstract}
We study the effect of varying wiring in excitable random networks in which connection weights change with activity to mold local resistance or facilitation due to fatigue. Dynamic attractors, corresponding to patterns of activity, are then easily destabilized according to three main modes, including one in which the activity shows chaotic hopping among the patterns. We describe phase transitions to this regime, and show a monotonous dependence of critical parameters on the heterogeneity of the wiring distribution. Such correlation between topology and functionality implies, in particular, that tasks which require unstable behavior - such as pattern recognition, family discrimination and categorization - can be most efficiently performed on highly heterogeneous networks. It also follows a possible explanation for the abundance in nature of scale-free network topologies.
\end{abstract}

PACS numbers: 64.60.an, 05.45.-a, 84.35.+i, 87.19.lj

Excitable systems allow for the regeneration of waves propagating through them, and may thus respond vigorously to weak stimulus. The brain and other parts of the nervous system are wellstudied paradigms, and forest fires with constant ignition of trees and autocatalytic reactions in surfaces, for instance, also share some of the basics $(1 ; 2 ; 3 ; 4 ; 5)$. The fact that signals are not gradually damped by friction in these cases is a consequence of cooperativeness between many elements in a nonequilibrium setting. In fact, the systems of interest may be viewed as large networks whose nodes are "excitable". This, which admits various realizations, typically means that each element has a threshold and a refractory time between consecutive responses.

Brain tasks may ideally be reproduced in mathematical neural networks. These consist of neurons - often simplified as binary variables - connected by edges representing synapses ( 6 ; 7; 8). Assuming edges weighted according to a prescription (e.g. (9)) which in a sense saves information from a set of given patterns of activity, these patterns become attractors of the phasespace dynamics. Therefore, the system shows retrieval of the stored patterns, known as associative memory. Actual neural systems do much more than just recalling a memory and staying there, however. That is, one should expect dynamic instabilities or other destabilizing mechanism. This expectation is reinforced by recent experiments suggesting that synapses undergo rapid changes with time which may both determine brain tasks $(10 ; 11 ; 12 ; 13)$ and induce irregular and perhaps chaotic activity $(14 ; 15)$.

The observed rapid changes (which have been described $(11 ; 12 ; 13$ ) as causing "synaptic depression" and/or "facilitation" on the time scale of milliseconds -i.e., much faster than the plasticity process in which synapses store patterns) may correspond to the characteristic behavior of single excitable elements. Furthermore, a fully-connected network which describes cooperation between such excitable elements exhibits both attractors and chaotic instabilities (16). Here, we extend and generalize this study to conclude on the influence of the excitable network topology on dynamic behavior. We show, in particular, an interesting correlation between certain wiring topology and optimal functionality.

Consider $N$ binary nodes, $s_{i}= \pm 1$, and the topology matrix, $\epsilon_{i j}=1$, 0 , which indicates the existence or not of an edge between nodes $i, j=1,2, \ldots, N$. Let a set of $M$ patterns, $\xi_{i}^{\nu}= \pm 1$, $\nu=1, \ldots M$ (which we generate here at random), and assume that they are "stored" by giving 
each edge a base weight $\overline{\omega_{i j}}=N^{-1} \sum_{\nu} \xi_{i}^{\nu} \xi_{j}^{\nu}$. Actual weights are dynamic, however, e.g., $\omega_{i j}=$ $\overline{\omega_{i j}} x_{j}$ where $x_{j}$ is a stochastic variable. Assuming the limit in which this varies in a time scale infinitely smaller than the one for node dynamics, we may consider a stationary distribution such as $P\left(x_{j} \mid S\right)=q \delta\left(x_{j}-\Xi_{j}\right)+(1-q) \delta\left(x_{j}-1\right), S=\left\{s_{j}\right\}$, for instance. This amounts to assume that, at each time step, every connection has a probability $q$ of altering its weight by a factor $\Xi_{j}$ which is a function (to be determined) of the local field at $j$, namely, the net current from other nodes. This choice differs essentially from the one in Ref.(16), where $q$ depends on the global degree of order and $\Xi_{j}$ is a constant independent of $j$.

Assume independence of the noise at different edges, and that the transition rate for the stochastic changes is

$$
\frac{\bar{c}\left(S \rightarrow S^{i}\right)}{\bar{c}\left(S^{i} \rightarrow S\right)}=\prod_{j / \epsilon_{i j}=1} \frac{\int d x_{j} P\left(x_{j} \mid S\right) \Psi\left(u_{i j}\right)}{\int d x_{j} P\left(x_{j} \mid S^{i}\right) \Psi\left(-u_{i j}\right)},
$$

where $u_{i j} \equiv s_{i} s_{j} x_{j} \overline{\omega_{i j}} T^{-1}, \Psi(u)=\exp \left(-\frac{1}{2} u\right)$ to have proper contour conditions, $T$ is a "temperature" parameter, and $S^{i}$ stands for $S$ after the change $s_{i} \rightarrow-s_{i}$. (For a description of this formalism and its interpretation, see (17).) We define the effective local fields $h_{i}^{\mathrm{eff}}=h_{i}^{\mathrm{eff}}(S, T, q)$ via $\prod_{j} \varphi_{i j}^{-} / \varphi_{i j}^{+}=\exp \left(-h_{i}^{\text {eff }} s_{i} / T\right)$, where $\varphi_{i j}^{ \pm} \equiv q \exp \left( \pm \Xi_{j} v_{i j}\right)+(1-q) \exp \left( \pm v_{i j}\right)$, with $v_{i j}=\frac{1}{2} \epsilon_{i j} u_{i j}$. Effective weights $\omega_{i j}^{\text {eff }}$ then follow from $h_{i}^{\text {eff }}=\sum_{j} \omega_{i j}^{\text {eff }} s_{j} \epsilon_{i j}$. To obtain an analytical expression, we linearize around $\overline{\omega_{i j}}=0$ (a good approximation when $\left.M \ll N\right)$, which yields

$$
\omega_{i j}^{\mathrm{eff}}=\left[1+q\left(\Xi_{j}-1\right)\right] \overline{\omega_{i j}} .
$$

In order to fix $\Xi_{j}$ here, we first introduce the overlap vector $\vec{m}=\left(m^{1}, \ldots m^{M}\right)$, with $m^{\nu} \equiv$ $N^{-1} \sum_{i} \xi_{i}^{\nu} s_{i}$, which measures the correlation between the current configuration and each of the stored patterns, and the local one $\vec{m}_{j}$ of components $m_{j}^{\nu} \equiv\langle k\rangle^{-1} \sum_{l} \xi_{l}^{\nu} s_{l} \epsilon_{j l}$, where $\langle k\rangle$ is the mean node connectivity, i.e., the average of $k_{i}=\sum_{j} \epsilon_{i j}$. We then assume, for any $q \neq 0$, that the factor is $\Xi_{j}=1+\zeta\left(h_{j}^{\nu}\right)(\Phi-1) / q$, with

$$
\zeta\left(h_{j}^{\nu}\right)=\chi^{\alpha} /(1+M / N) \sum_{\nu}\left|h_{j}^{\nu}\right|^{\alpha}
$$

where $\chi \equiv N /\langle k\rangle$ and $\alpha>0$ is a parameter. This comes from the fact that the field at node $j$ may be written as a sum of components from each pattern, namely, $h_{j}=\sum_{\nu}^{M} h_{j}^{\nu}$, where

$$
h_{j}^{\nu}=\xi_{j}^{\nu} N^{-1} \sum_{i} \epsilon_{i j} \xi_{i}^{\nu} s_{i}=\chi^{-1} \xi_{j}^{\nu} m_{j}^{\nu}
$$

Our choice for $\Xi_{j}$, which amounts to assume that the "fatigue" at a given edge increases with the field at the preceding node $j$ (and allows to recover the fully-connected limit in (16) if $\alpha=2$ ), finally leads to

$$
\omega_{i j}^{\mathrm{eff}}=\left[1+(\Phi-1) \zeta_{j}\left(\overrightarrow{m_{j}}\right)\right] \overline{\omega_{i j}} .
$$

Varying $\Phi$ one sets the nature of the weights. That is, $0<\Phi<1$ corresponds to resistance (depression) due to heavy local work, while the edge facilitates, i.e., tends to increase the effect of the signal under the same situation for $\Phi>1$. (The action of the edge is reversed for negative $\Phi$.) We performed Monte Carlo simulations using standard parallel updating with the effective rates $\bar{c}\left(S \rightarrow S^{i}\right)$ computed using the latter effective weights.

It is possible to solve the single pattern case $(M=1)$ under a mean-field assumption, which is a good approximation for large enough connectivity. That is, we may substitute the matrix $\epsilon_{i j}$ 
by its mean value over network realizations to obtain analytical results that are independent of the underlying disorder. Imagine that each node hosts $k_{i}$ half-edges according to a distribution $p(k)$, the total number of half-edges in the network being $\langle k\rangle N$. Choose a node $i$ at random and randomly join one of its half-edges to an available free half-edge. The probability that this halfedge ends at node $j$ is $k_{j} /(\langle k\rangle N)$. Once all the nodes have been linked up, the expected value (as a quenched average over network realizations) for the number of edges joining nodes $i$ and $j$ is $\overline{\epsilon_{i j}}=k_{i} k_{j} /(\langle k\rangle N)$ (18). Using the notation $\eta_{i} \equiv \xi_{i} s_{i}$, we have $m_{j}=\chi\left\langle\eta_{i} \epsilon_{i j}\right\rangle_{i}=\frac{\chi}{N} \sum_{i} \eta_{i} \epsilon_{i j}$. Because node activity is not statistically independent of connectivity (21), we must define a new set of overlap parameters, analogous to $m$ and $m_{j}$. That is, $\mu_{n} \equiv\left\langle k_{i}^{n} \eta_{i}\right\rangle_{i} /\left\langle k^{n}\right\rangle$ and the local versions $\mu_{n}^{j} \equiv \chi\left\langle k_{i}^{n} \eta_{i} \epsilon_{i j}\right\rangle_{i} /\left\langle k^{n}\right\rangle$. After using $\epsilon_{i j}=\overline{\epsilon_{i j}}$, one obtains the relation $\mu_{n}^{i}=\left\langle k^{n+1}\right\rangle k_{i} \mu_{n+1} /\left(\left\langle k^{n}\right\rangle\langle k\rangle^{2}\right)$. Inserting this expression into the definition of $\mu_{n}$, and substituting $\left\langle s_{i}\right\rangle=\tanh \left[T^{-1} h_{i}^{\text {eff }}(S)\right]$ (for very large $\mathrm{N}$ ), standard mean-field analysis yields

$$
\mu_{n}(t+1)=\frac{1}{\left\langle k^{n}\right\rangle}\left\langle k^{n} \tanh M_{T, \Phi}(k, t)\right\rangle_{k},
$$

where the last quantity is defined as

$$
M_{T, \Phi}=\frac{k}{T N}\left[\mu_{1}(t)+(\Phi-1) \frac{\left\langle k^{\alpha+1}\right\rangle}{\langle k\rangle^{\alpha+1}}\left|\mu_{1}(t)\right|^{\alpha} \mu_{\alpha+1}(t)\right] .
$$

This is a two-dimensional map which is valid for any random topology of distribution $p(k)$. Note that the macroscopic magnitude of interest is $\mu_{0}=m \equiv|\vec{m}|$.

A main consequence of this is the existence of a critical temperature, $T_{c}$, under very general conditions, e.g., for many different network connectivities. More specifically, as $T$ is decreased, the overlap $m$ describes a second-order phase transition from a disordered or, say, "paramagnetic" phase to an ordered ("ferromagnetic") phase which exhibits associative memory. The mean-field temperature signaling this transition is

$$
T_{c}=\left\langle k^{2}\right\rangle(\langle k\rangle N)^{-1} .
$$

On the other hand, the map reduces for $T=0$ to $\mu_{n}(t+1)=$ $\operatorname{sign}\left\{\mu_{n}(t)\left[1+(\Phi-1)\langle k\rangle^{\alpha+1} /\left\langle k^{\alpha+1}\right\rangle\right]\right\}$. This implies the existence at $\Phi=\Phi_{0}$, where

$$
\Phi_{0}=1-\langle k\rangle^{\alpha+1} /\left\langle k^{\alpha+1}\right\rangle,
$$

of a transition as $\Phi$ is decreased from the ferromagnetic phase to a new phase in which periodic hopping between the attractor and its negative occurs. This is confirmed by the Monte Carlo simulations for $M>1$, namely, the hopping is also among different attractors for finite $T$. The simulations also indicate that this transition washes out at low enough finite temperature. Instead, actual evolutions show that, for a certain range of $\Phi$ values, the system activity then exhibits chaotic behavior.

The transition from ferromagnetic to chaotic states is a main concern hereafter. Our interest in this regime follows from several recent observations concerning the relevance of chaotic activity in a network. In particular, it has been shown that chaos might be responsible for certain states of attention during brain activity $(22 ; 23)$, and that some network properties such as the computational capacity (24) and the dynamic range of sensitivity to stimuli (25) may become optimal at the "edge of chaos" in a variety of settings.

We next notice that the critical values $T_{c}$ and $\Phi_{0}$ only depend on the moments of the generic distribution $p(k)$, and that the ratio $\left\langle k^{a}\right\rangle /\langle k\rangle^{a}, a>1$, is a convenient way of characterizing heterogeneity. We studied in detail two particular types of connectivity distributions with easily 
tunable heterogeneity, namely, networks with $\langle k\rangle N / 2$ edges randomly distributed with $p(k)$ such that the heterogeneity depends on a single parameter. Our first case is the bimodal distribution, $p(k)=\frac{1}{2} \delta\left(k-k_{1}\right)+\frac{1}{2} \delta\left(k-k_{2}\right)$ with parameter $\Delta=\left(k_{2}-k_{1}\right) / 2=\langle k\rangle-k_{1}=k_{2}-\langle k\rangle$. Our second case is the scale-free distribution, $p(k) \sim k^{-\gamma}$, which does not have any characteristic size but $k$ is confined to the limits, $k_{0}$ and $k_{m} \leq \min \left(k_{0} N^{\frac{1}{\gamma-1}}, N-1\right)$ for finite $N(26)$. Notice that the network in this case gets more homogeneous as $\gamma$ is increased (27), and that this kind of distribution seems to be most relevant in nature $(20 ; 21 ; 28 ; 29)$. In particular, the functional topology in the human brain, as defined by correlated activity between small clusters of neurons, has been shown to correspond to this case with exponent $\gamma \simeq 2(\underline{30})$ (in spite of the fact that the brain's structural or wiring topology is not yet well known).

We obtained the critical value of the fatigue, $\Phi_{c}(T)$, from Monte Carlo simulations at finite temperature $T$. These indicate that chaos never occurs for $T \gtrsim 0.35 T_{c}$. On the other hand, a detailed comparison of the value $\Phi_{c}$ with $\Phi_{0}$ - as obtained analytically for $T=0$ - indicates that $\Phi_{c} \simeq \Phi_{0}$. Figure 1 illustrates the "error" $\Phi_{0}-\Phi_{c}(T)$ for different topologies. This shows that the

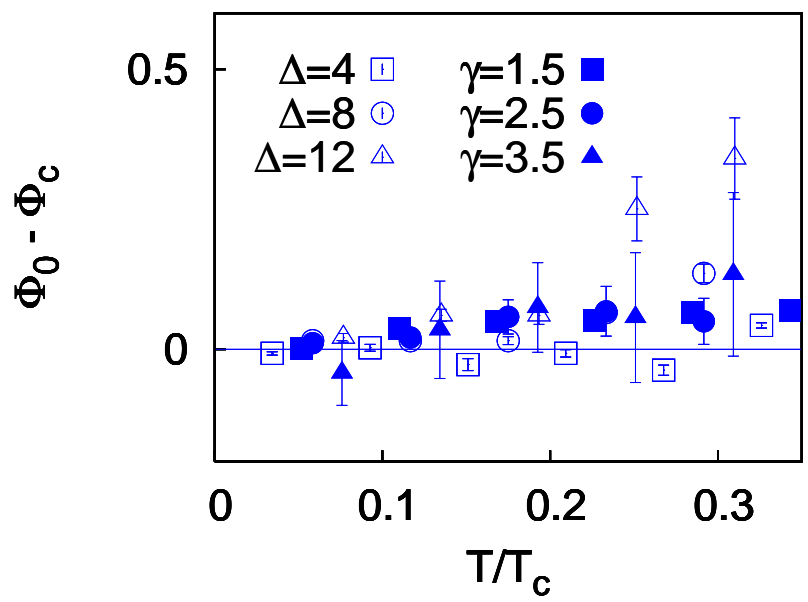

FIG. 1 The temperature dependence of the difference between the values for the fatigue at which the ferromagnetic-periodic transition occurs, as obtained analytically for $T=0\left(\Phi_{0}\right)$ and from MC simulations at finite $T\left(\Phi_{c}\right)$. The critical temperature is calculated as $T_{c}=\left\langle k^{2}\right\rangle(\langle k\rangle N)^{-1}$ for each topology. Data are for bimodal distributions with varying $\Delta$ and for scale-free topologies with varying $\gamma$, as indicated. Here, $\langle k\rangle=20, N=1600$ and $\alpha=2$. The bars are standard deviations for 10 network realisations.

approximation $\Phi_{c} \simeq \Phi_{0}$ is quite good at low $T$ for any of the cases examined. Therefore, assuming the critical values for the main parameters, $T_{c}$ and $\Phi_{0}$, as given by our map, we conclude that the more heterogeneous the distribution of connectivities of a network is, the lower the amount of fatigue, and the higher the critical temperature, needed to destabilize the dynamics. As an example of this interesting behavior, consider a network with $\langle k\rangle=\ln (N)$, and dynamics according to $\alpha=2$. If the distribution were regular, the critical values would be $T_{c}=\ln (N) / N$ (which goes to zero in the thermodynamic limit) and $\Phi_{0}=0$. However, a scale-free topology with the same number of edges and $\gamma=2$ would yield $T_{c}=1$ and $\Phi_{0}=1-2(\ln N)^{3} / N^{2}$ (which goes to 1 as $N \rightarrow \infty$ ).

Figure 2 illustrates, for two topologies, the phase diagram of the ferromagnetic-chaotic transition. Most remarkable is the plateau observed in the edge-of-chaos or transition curve for scale-free topologies around $\gamma \simeq 2$, for which very little fatigue, namely, $\Phi \lesssim 1$ which corresponds to slight depression, is required to achieve chaos. The limit $\gamma \rightarrow \infty$ corresponds to $\langle k\rangle$-regular graphs (equivalent to $\Delta=0$ ). If $\gamma$ is reduced, $k_{m}$ increases and $k_{0}$ decreases. The network is truncated 


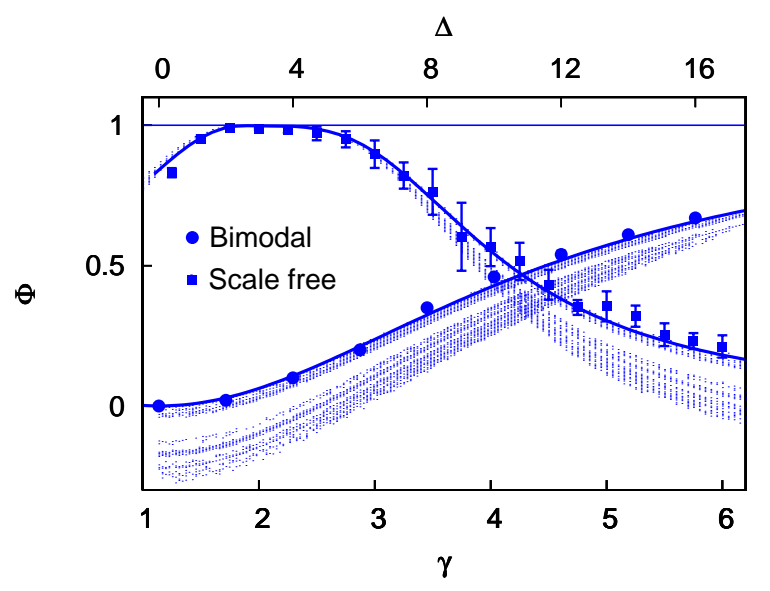

FIG. 2 The critical fatigue values $\Phi_{0}$ (solid lines) and $\Phi_{c}$ from MC averages over 10 networks (symbols) with $T=2 / N,\langle k\rangle=20, N=1600, \alpha=2$. The dots below the lines correspond to changes of sign of the Lyapunov exponent as given by the iterated map, which roughly agree with the other results. This is for bimodal and scale-free topologies, as indicated.

when $k_{m}=N$. It follows that a value of $\gamma$ exits at which $k_{0}$ cannot be smaller, so that $k_{m}$ must drop to preserve $\langle k\rangle$. This explains the fall in $\Phi_{c}$ as $\gamma \rightarrow 1$.

As a further illustration of our findings, we monitored the performance as a function of topology during a simulation of pattern recognition. That is, we "showed" the system a pattern, say $\nu$ chosen at random from the set of $M$ previously stored, every certain number of time steps. This was performed in practice by changing the field at each node for one time step, namely, $h_{i} \rightarrow h_{i}+\delta \xi^{\nu}$, where $\delta$ measures the intensity of the input signal. Ideally, the network should remain in this configuration until it is newly stimulated. The performance may thus be estimated from a temporal average of the overlap between the current state and the input pattern, $\left\langle m^{\nu}\right\rangle_{\text {time }}$. This is observed to simply increase monotonically with $\Delta$ for the bimodal case. The scale-free case, however, as illustrated in figure 3, shows how the task is better performed the closer to the edge of chaos the

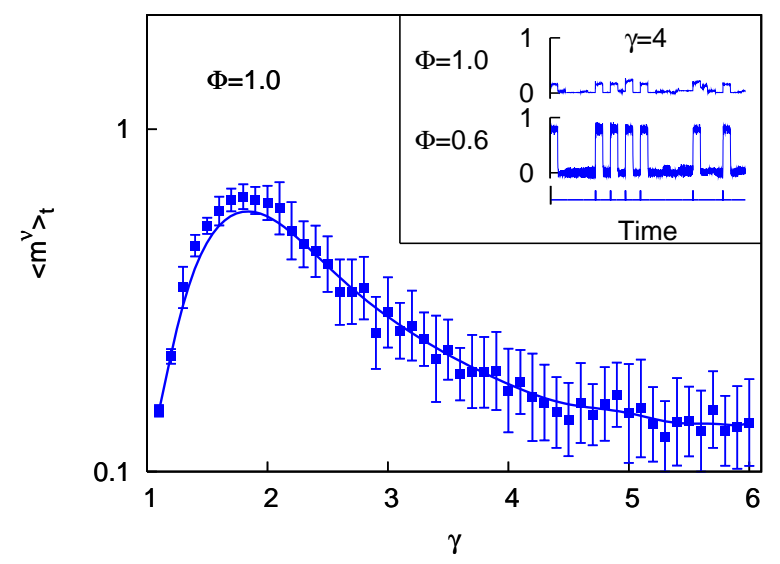

FIG. 3 Network "performance" (see the main text) against $\gamma$ for scale-free topology with $\Phi=1$, as an average over 20 network realizations with stimulation every $50 \mathrm{MC}$ steps for $2000 \mathrm{MC}$ steps, $\delta=5$ and $M=4$; other parameters as in Fig. 2. Inset shows sections of typical time series of $m^{\nu}$ for $\gamma=4$; the corresponding stimulus for pattern $\nu$ is shown below.

network is. This is because the system is then easily destabilized by the stimulus while being 
able to retrieve a pattern with accuracy. Figure 3 also shows that the best performance for the scale-free topology when $\Phi=1$, i.e., in the absence of any fatigue, definetely occurs around $\gamma=2$.

The fact that the model network above is one of the simplest situations one may conceive with dynamic connections which depend on local fields - suggest that topological heterogeneity may indeed be a relevant property for a complex network to perform efficiently certain high level functions. This has in practice been illustrated before using networks with a similar philosophy which happen to be useful for pattern recognition and class identification (31). Our system retrieves memory patterns with accuracy in spite of noise, and yet it may easily destabilize itself to change state in response to an input signal - without requiring an excessive fatigue for the purpose. There is a correlation between the amount $\Phi$ of fatigue and the value of $\gamma$ for which performance is maximized. One may argue that the plateau of "good" behavior shown around $\gamma \simeq 2$ for scale-free networks with $\Phi \lesssim 1$ (figure 22) is a possible justification for the supposed tendency of certain systems in nature to evolve towards this topology. It may also serve as a hint when implementing artificial networks.

We thank M.A. Muñoz for very helpful comments. This work was financed by the JA project FQM-01505 and by the MEC-FEDER project FIS2005-00791.

\section{References}

[1] P. Bak et al., Phys. Lett. A 147, 297 (1990)

[2] E. Meron, Phys. Rep. 218, 1 (1992)

[3] B. Lindner et al., Phys. Rep. 392, 321 (2004).

[4] E. M. Izhikevich, Dynamical Systems in Neuroscience, MIT Press, Cambridge MA 2007.

[5] A. Arenas et al., Rev. Mod. Phys. (2008)

[6] S. Amari, IEEE Trans. Syst. Man. Cybern. 2, 643 (1972)

[7] J.J. Hopfield, Proc. Natl. Acad. Sci. USA 79, 2554 (1982)

[8] D.J. Amit, Modeling Brain Function, Cambridge Univ. Press, 1989

[9] D.O. Hebb, The Organization of Behavior, Wiley, New York 1949

[10] L.F. Abbott et al., Science 275, 220 (1997)

[11] M.V. Tsodyks et al, Neural Comp. 10, 821 (1998)

[12] R.C. Malenka and R.A. Nicoll, Science 285, 1870 (1999)

[13] L. Pantic et al., Neural Comp. 14, 2903 (2002)

[14] J.M. Barrie et al., J. Neurophysiol. 76, 520 (1996)

[15] H. Korn and P. Faure, C. R. Biol. 326, 787 (2003)

[16] J. Marro et al., J. Stat. Mech. P02017 (2008)

[17] J. Marro and R. Dickman, Nonequilibrium Phase Transitions in Lattice Models, Cambridge Univ. Press, 1999.

[18] Assuming one edge at most between any two nodes, $\epsilon_{i j}=0,1$, the value will be slightly smaller, but it is easy to prove that this is also a good approximation for $k_{i}, k_{j} \ll N$. If more than one edge is permitted, which is more realistic, this result is exact for any random network, and it agrees (for a power-law distribution with $\gamma=3$ ) with the one obtained in (19) for an evolving network (20).

[19] G. Bianconi, G., Phys. Lett. A 303, $116(2002)$

[20] A.-L Barabási and R. Albert, Science 286, 509 (1999)

[21] J.J. Torres et al., Neurocomputing 58-60, 229 (2004)

[22] J.J. Torres et al., Int. J. Bifur. Chaos (2008)

[23] J.J. Torres et al., Neural Nets., submitted.

[24] N. Bertschinger and T. Natschläger, Neural Comp. 16, 1413 (2004)

[25] V.R.V. de Assis and M. Copelli, preprint arXiv: 0707.0309-q-bio.

[26] Though the minimum connectivity is 1 , we shall assume $k_{0} \geq 2$, which reduces the probability of disconnection.

[27] The distribution is truncated and therefore not strictly scale free for $\gamma<2$. However, nature shows examples for which $\gamma$ is slightly larger than 1 , so that we consider the whole range here. 
[28] M.E.J. Newman, SIAM Reviews 45, 167 (2003)

[29] S. Boccaletti et al., Phys. Rep. 424, 175 (2006)

[30] V.M. Eguíluz et al., Phys. Rev. Lett. 94, 018102 (2005)

[31] J.M. Cortes et al., AIP Conf. Proc. 779, 178 (2005) 